capsule of the kidney in forming permanent anchorage by pararenal adhesions. For years I have placed more reliance on the tampon than sutures in the fixation of a movable kidney. My present method of nephropexy consists in exposing the kidney by Simon's vertical lumbar incision. As soon as the adipose capsule is reached the kidney is placed in proper position and is pushed forward into the wound by an assistant. About half of the kidney should project below the lower margin of the last rib. With dissecting forceps and curved scissors the adipose capsule is excised over the whole posterior surface of the kidney. The kidney is now brought well forward into the wound, the cut margins of the adipose capsule are pushed away from the kidney until the borders are freely exposed, when the fibrous capsule is thoroughly scarified with a long needle.

At this stage of the operation the lower pole of the kidney is grasped by its capsule with a French vulsellum forceps and brought well forward into the wound. With dissecting forceps, finger and blunt dissector the lower third of the kidney is laid bare and a strip of iodoform gauze about an inch in width and composed of four layers of gauze, is placed underneath the lower end of the kidney and each end brought out over the respective wound margin. By making traction on the forceps and gauze strip the lower end of the kidney is brought sufficiently forward to rest in the lower angle of the external incision. During the operation the margins of the external incision must be well retracted. With a long strip of iodoform gauze the floor of the wound is then carefully packed in such a way as to force the pararenal fat away from the borders of the kidney, leaving the posterior scarified surface of the kidney well exposed, when, with the same strip of gauze, this is covered and the whole wound well tamponed with another piece of gauze. The strip of gauze holding the kidney. is then tied over the iodoform gauze tampon, which forms a wedge, and will effectually prevent displacement of the organ until firm adhesion has rendered any direct mechanical support superfluous. The two pieces of gauze are tied together and the wound dressed in the usual manner. No part of the lumbar incision is sutured. The patient is then placed upon the back and a firm compress the size of an adult's fist is placed over the kidney below the costal arch and held in place by a wide strip of adhesive plaster encircling the entire body. The patient is placed in bed with the pelvis slightly elevated, and is directed to remain in the dorsal recumbent position or side operated upon for at least four weeks, the time required for the formation of pararenal adhesions suff. ciently firm to hold the organ permanently and securely in its new location. At the end of five or six days the tampon is removed. At this time the whole wound, including the capsule of the kidney will be found paved with vigorous granulations. The granulating surfaces are now brought together and held in contact with strips of adhesive plaster over a small absorbent aseptic dressing. Over this an additional dressing is applied, which with the abdominal compress is held in place with an additional strip of adhesive plaster and gauze roller. At the end of three or four weeks the whole wound will be found healed by primary intention and the kidney firmly fixed in its new location. The retention of the kidney in its normal location by this method affords not only a firm support by permanently fixing the lower end in the lower angle of the external incision and by securing broad surface adhesions, but the oblique angle in which it is anchored adds another mechanical condition calculated to maintain the organ in position, while this position will also tend to correct flexion of the ureter if such exists at the time the operation is performed. The immediate and remote results obtained by this method of operating in the last four cases have proved so satisfactory that I am not disposed to return to suturing again and strongly recommend this method of performing nephropexy for further trial by the profession.

\section{THE TECHNIQUE OF ABDOMINAL. HYSTERECTOMY.}

Presented to the Section on Obstetrics and Diseases of Women at the Forty-eighth Annual Meeting of the American Medical Association, held at P'hiladelphia, Pa., June 1-4, 1897. BY J. F. BALDWIN, A.M., M.D.,

PROFEBSOR OF OPERATIVE GYNECOLOGY, OHIO MEDICAI. UNIVERSITY; GYNECOLOGIST TO PROTESTANT HOSPITAL, ETC.

COLUMBUS. OHIO.

The abdomen is to be opened in the usual way and by as free an incision as necessary. Free the uterine mass from adhesions as thoroughly as possible, and draw it up into the incision; draw up the most accessible broad ligament and clamp it just outside of the ovary with a long clamp, the point of which is directed obliquely downward toward the cervix; with a short forceps grasp the upper border of the broad ligament near the uterus, so as to prevent recurrent hemorrhage through the ovarian artery; then sever the broad ligament with scissors, along the clamp first applied. The other broad ligament is then, if possible, to be treated in the same way. A peritoneal flap is now made in front, between the tips of the two clamps; this is best done with scissors; as this flap is dissected up, the bladder is carried with it and is thus separated from the uterus; a shorter peritoneal flap is then made on the posterior surface between the tips of the clamps.

Up to this point the uterus itself has not been interfered with, and the uterine arteries have not yet been reached. The layers of the broad ligament are now separated on each side, by the fingers, between the tip of the clamp on each side and the uterus, and the uterine arteries found and seized with forceps. With scissors curved on the flat (or, better still, with a Kelly's spud), the body of the uterus is next de. tached at or just below the level of the internal os, being so detached as to give an anterior and posterior flap of uterine tissue. In case there is any great difficulty in reaching the uterine artery before detaching the uterus itself, its seizure may be omitted until it spurts, as the uterus is being severed, when it is easily seized by an assistant. It is sometimes difficult, or even impossible, to secure both arteries before getting the tumor out of the way. In that case, I cut across from the side on which I have seized the artery and, after separating the mass, seize the base of the opposite broad ligament en masse below the point of separation, and then enucleate the tumor from below upward. This is the method recommended by Kelly, and one which, in suitable cases, possesses points of advantage.

The tumor has now been removed, the pelvis is empty, and hemorrhage has been entirely controlled, except perhaps a little oozing from the cervical flaps. If the patient has not already been placed in the 
Trendelenburg position, this should now be done. brought into apposition by a running suture. This The uterine arteries are next separated from any sur- suture is first inserted through the anterior cervical rounding tissue and ligated as far back as possible, flap at one side, is then passed through the end of the with fine silk, catgut or kangaroo tendon, and the pro- round ligament of that side, and then through the posjecting ends snipped off. The round ligament is then terior flap. As this suture is now made taut and tied, caught with forceps next to the long clamp, so as to the round ligament becomes implanted between the enable it to be quickly found later on, and the clamp flaps. By a similar maneuver the opposite round removed from the broad ligament. The ovarian artery ligament, at the proper time, is implanted between the is then easily found and drawn out from between the flaps upon that side. If the round ligaments are too folds of the ligament, ligated and the projecting end long they can be shortened before being transfixed, cut off. The same is then done on the opposite side. so as to give the desired degree of support to the Four ligatures have controlled all hemorrhage, though stump.

occasionally an aberrant branch will require ligation. Commencing next at the upper edge of one broad

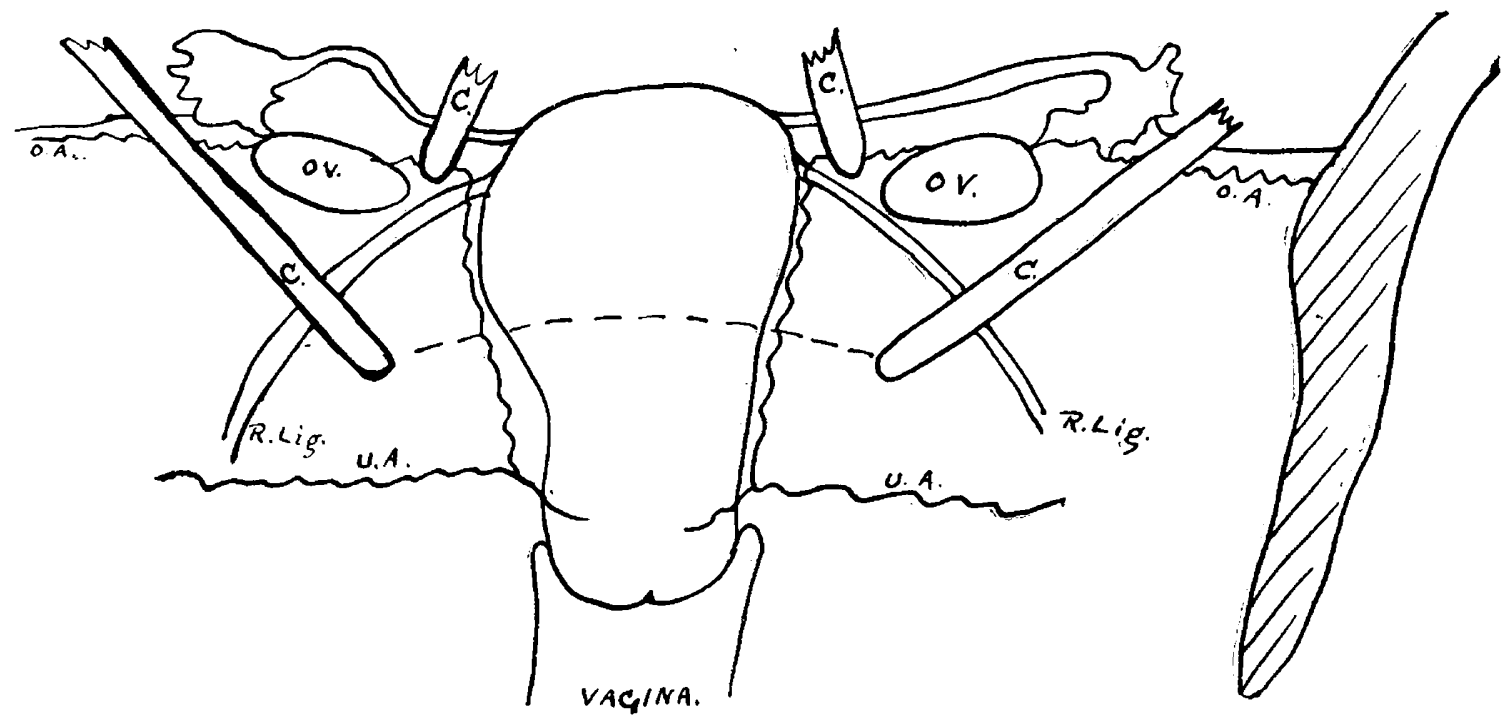
Diagram of operation, front view.-C., C., C., C., clamps; O., A., ovarian artery; Ov., ovary; U. A., uterine artery.
Broken line represen ts peritoneal flap.

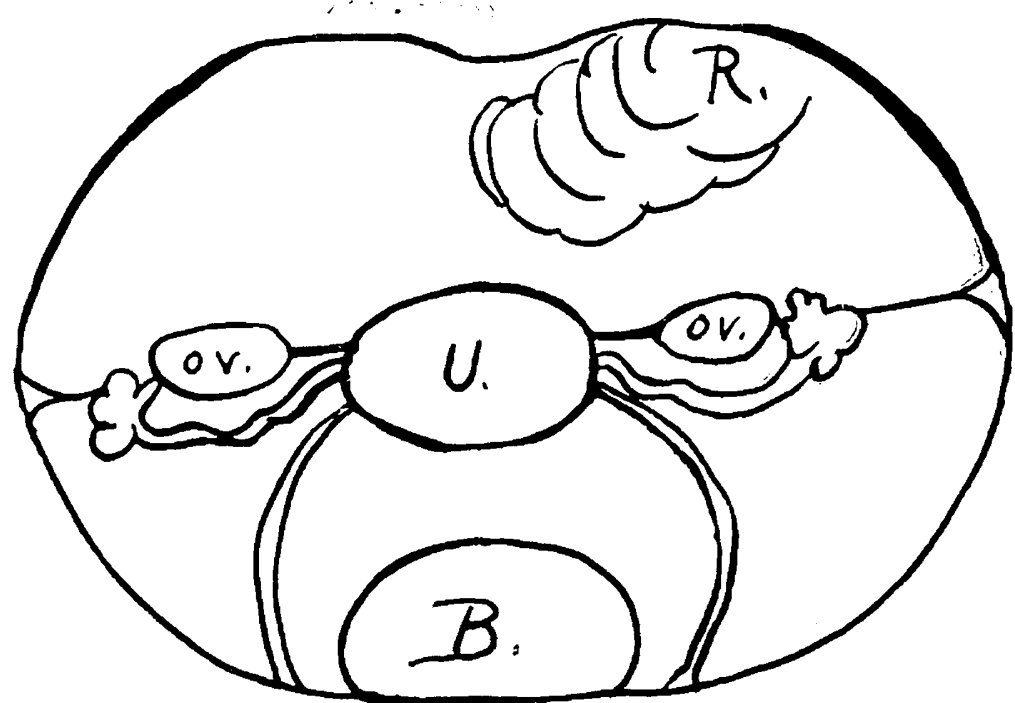

Diagram of pelvis seen from above.-B., bladder; Ov., ovary; R., rectum; U., uterus.

A long probe is now threaded with a strip of iodoform ligament stump, the peritoneal layers are inverted gauze, about one inch wide, passed through the cervical and with kangaroo tendon, or catgut, are united by canal into the vagina, and seized by an assistant. an "over-and-over" suture which runs down the The gauze is drawn through the cervix, except an broad ligament, then across over the cervical stump, inch or two which I hold in my fingers, and the probe detached as it appears at the vulva. The end of the gauze which projects above is now cut off just flush with the bottom of the wound. The passage of this gauze mechanically cleans the cervical canal, and also secures drainage in case of any oozing.

turning in the peritoneal flaps as it proceeds, and up on the opposite side. This suture should be so inserted as to draw the tissues of the broad ligament snugly together. These being thus snugly apposed, unite so as to make excellent supports for the stump in addition to the round ligaments already attached.

With kangaroo tendon the uterine flaps are now The operation when thus completed leaves a perfectly 
smooth pelvic floor, with, at no point, the exposure of any raw surface for adhesions.

In case, for any reason, drainage of the pelvis is desired, this is accomplished by opening Douglas's cul-de-sac and introducing a strip of gauze into the vagina. The abdomen is then closed in the usual way. My own method is to unite the fascia, muscle and peritoneum with silver wire, the twisted ends being
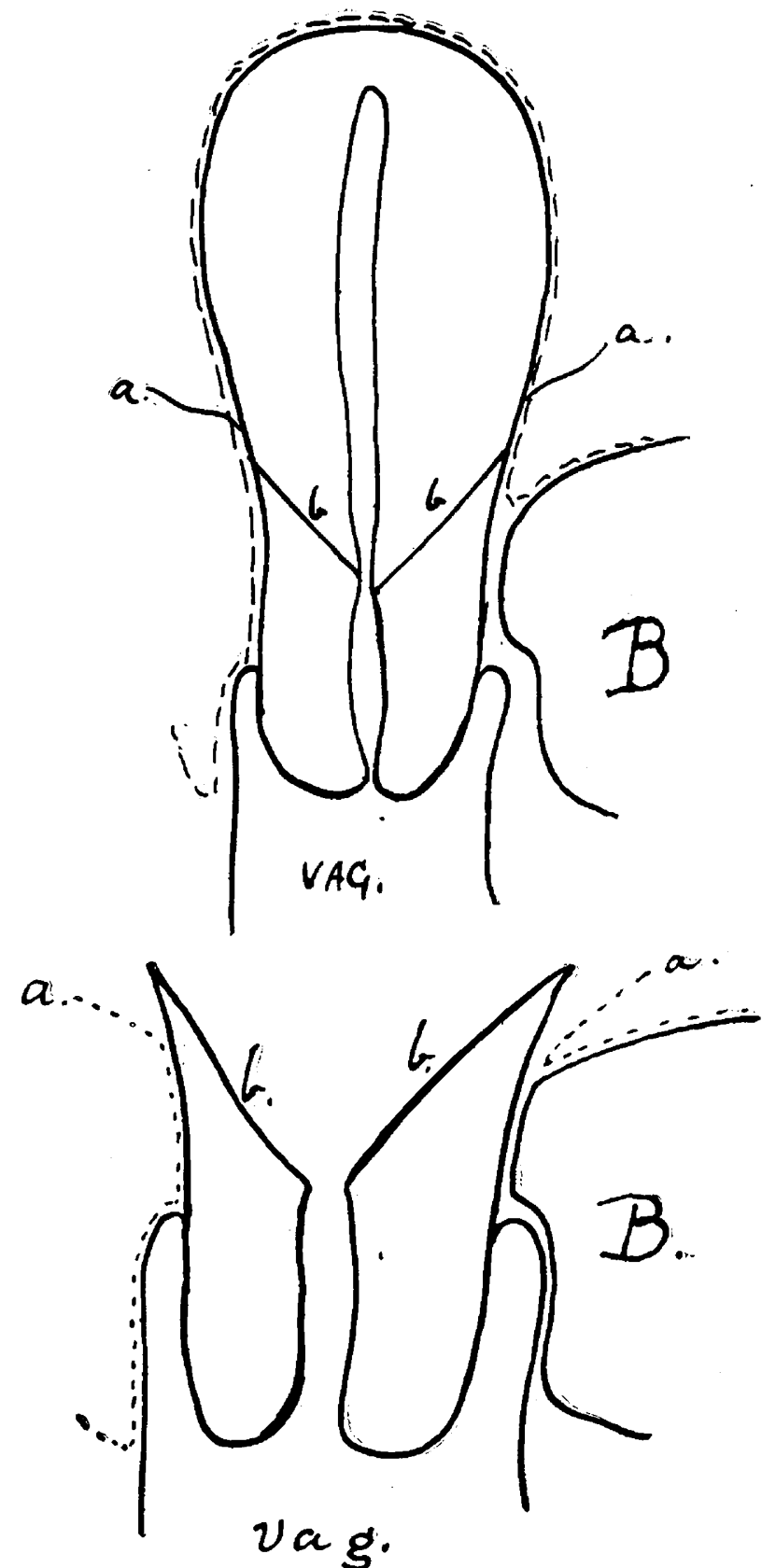

a, a, points of making peritoneal flaps; b, b, lines of amputation of cervix. Dotted line represents peritoneum.

cut short and turned down. As it has been found that in about one case in twenty some of these wires become infected and give rise to sinuses which remain until the wires are removed, I have for several months used another method of closing the incision; silk-worm gut sutures are first introduced, these passing through the entire thickness and including plenty of fascia and muscle, but only a little of the skin and peritoneum. The fascia is then approximated by a continuous suture of silver wire, the ends of which emerge about an inch beyond the ends of the incision. Before tightening the wire, the silk-worm gut sutures are drawn up snugly and held by an assistant, but not tied; the wire is then seized at each end, drawn taut, and pulled back and forth until all bends are removed and it is practically straight; the ends are then fastened by perforated shot, several layers of iodoform gauze being interposed between the shot and the skin. The silk-worm gut sutures are then tied. The wire can be slipped out without any trouble at the end of ten days or two weeks. I am especially careful to get the fascia well into apposition. The skin is then closed with silkworm gut, or the subcuticular kangaroo tendon suture. If the abdominal walls have a thick layer of fat, this is held in apposition by two or three stay-sutures, as needed.

In case it is desirable to remove the entire uterus, the cervix instead of being cut across is enucleated in the usual way. It is sometimes better to remove the tumor first and then to enucleate the cervix. In such cases a pledget of gauze should be introduced into the vagina from above, so as to drain the point of separation of the cervix, but the peritoneum should be completely closed, as above described, including the bringing in of the round ligaments.

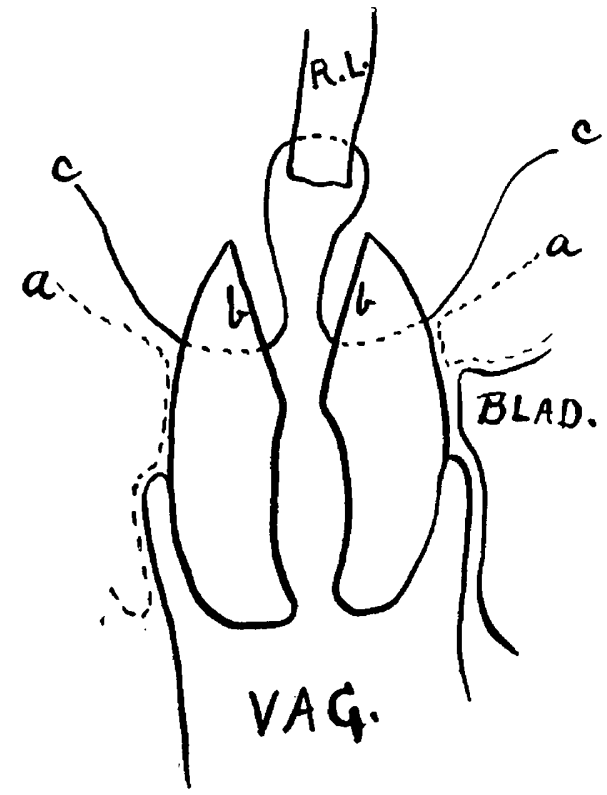

Dragram showing method of bringing down and fastening round ligaments in closing cervical flaps. a, a, peritoneal flaps; $b$, b, cervical flaps; c c. suture: R. L., round ligament.

During the last two and a half years in which I have been using this method, I have made ninetyfive operations with five deaths, a mortality of 5.26 per cent. Three of these were in cases of cancer of the fundus with wide dissection and unfavorable conditions. In a recent paper by E. W. Cushing, M.D., of Boston, on the "Evolution of Abdominal Hysterectomy," he tabulates 1,670 operations from the reports of thirty-six of the leading operators of the United States. The average mortality of these operaations was 13.8 per cent. During the last year I had the opportunity of watching abdominal hysterectomy as performed in many of the leading gynecologic clinics abroad. Almost without exception, I found their methods quite crude and unsystematic, while their mortality I found to vary from about 15 to 25 per cent.

In my own cases, in only one instance was death 
due to post-operative infection. Post-operative morbidity has been almost nil. Very few patients have remained in the hospital over twenty-one days after the operation. One case was removed to her own home on the tenth day. No cases of ventral hernia or of vaginal prolapse have occurred. There is sometimes some complaint of pain, but this is usually much less than after the performance of vaginal hysterectomy. Indeed, convalescence is very similar to that in normal child-bed, and patients usually sit up on the fourteenth day. The operation is a short one, usually requiring about forty minutes for its completion, and there is practically almost no hemorrhage. These two statements explain the fact that there is usually almost no shock.

The advantages of this method of operating are:

1. Such a shutting off of the vagina as to reduce to a minimum any danger of infection from that source.

2. The ligature placed around the uterine artery is entirely outside of the uterine wound, and being of fine material and buried in the tissues is much less likely to give any trouble.

3. The snug closing of the cervical tissue prevents oozing.

4. The smooth peritoneum in the floor of the pelvis, having no projecting stumps or raw surfaces, reduces to an absolute minimum the danger of intestinal adhesions.

$\tilde{0}$. The implantation of the round ligaments and puckering in of the stumps of the broad ligaments prevents prolapse of cervical stump and vagina.

6 . The use of the clamps on the broad ligaments obviates hemorrhage, leaves the parts in better shape for the subsequent steps of the operation, and saves considerable time.

\section{HOW SHALL WE FEED THE BABY?}

Presented to the Section on Diseases of Children, at the Forty-eighth Annual Meeting of the American Medical Association, held at Philadelphia, June 1-4, 1897.

BY A. C. COTTON, A.M., M.D. CHICAGO.

If at the breast and doing well, leave it alone. If not doing well, let us ascertain the cause. Does it present evidences of innutrition, malnutrition, acute indigestion or chronic indigestion? Is there starvation from insufficient or improper food, or starvation from repletion? In some cases the fault is apparent at a glance; in many it is quite difficult to determine at first wherein the trouble lies. The apparent error as to time, frequency of feeding and quantity having been corrected, the physician may finally have to face this question, "can this mother's milk be made to fill all the requirements of this babe?" and the sooner we ascertain just what and how much this mother is secreting, the sooner we are on the way to a solution of this problem. We must examine the breast milk as to its total amount and as to the relative quantity and quality of its constituents. The old haphazard procedures must be relegated to the past; the time has come when the same exactitude is demanded in this as in other means of diagnosis. We must know what the babe is yetting before we can intelligently consider any changes in food. Analysis, then, of the mother's milk must be the prime desideratum if we would give advice as to the feeding of babies.

There are a number of methods in vogue for determining, with greater or less accuracy, the relative pro- portion of some of the leading ingredients. For clin. ical purposes a determination of the total solids may be had from the known specific gravity, temperature and percentage of buster fat by means of Richmond's equation: $\mathrm{T}=(\mathrm{G} \div 4)+(6 \div 5) \mathrm{F}+0.14$, in which $\mathrm{T}=$ total solids, $G=$-specific gravity (the last two figures), and $\mathrm{F}=$ fat. Care should be taken to correct specific gravity for temperature if the ordinary hydrometer adjusted at 60 degrees $\mathrm{F}$. be employed. Holland subtracts one from the hydrometer reading for each ten degrees below 60 degrees $\mathbf{F}$., and adds one to the reading for each ten degrees above 60 degrees $\mathrm{F}$., proportionate fractions expressing variations of less than ten degrees. For determining the percentage of fat we have employed Fesser's lactoscope, also the methods of Holt, Marchand, Leffmann and Beam, and Babcock, with preference for the last-named on account of its simplicity and accuracy. The first mentioned has the disadvantage of inaccuracy from the varying visual power of different observers, also from the fact that the opacity of milk is not due alone to the fat globules but also to the phosphate of calcium present. Holt's method, so easy of employment, requires time, twenty-four hours, and the ratio 5 to 3 does not always express the relation of cream to fat. Marchand's test has never given the results obtained by us from more accurate analysis.

The centrifugal machines adapted to the Babcock test bottles have heretofore been too clumsy, but recently the Chicago Dairy Supply Company, No. 5 West Washington Street, has brought out a small cheap machine. My attention has been called to a bottle manfactured by Wood \& Comer, 130 Juvenal Street, Philadelphia, of shape and size to fit the ordinary office centrifuge and adapted by graduation to the Leffmann and Beam method. The capacity of this tube is certainly not more than 20 c.c., so that the quantity of milk required by the Leffmann and Beam test would not exceed 8 or 10 c.c., an item greatly in its favor. If experience proves the utility of this tube its advantages place it far in advance of any previous device for determining the fat in breast milk. The microscope is a necessary adjunct to the examination for the presence in the milk of blood, pus or colostrum corpuscles.

If the child's nutrition does not improve soon, I would remove it from the breast temporarily, keeping up lactation by pumping regularly and testing the milk meanwhile for the improvement sought. Not infrequently the child may be returned to the breast after an interval of from a few days to a few weeks. In my opinion there is no question as to the desirability of maternal nursing in preference to any method of artificial feeding. If, after all, the mother's milk can not be brought to suitable proportions for complete nutrition, or for any reason weaning is necessary, then substitute feeding must be adopted, and the choice of a substitute becomes at once a question of the highest importance. The multitude of infant foods and substitutes for mother's milk suggests the conclusion that none are completely successful, and the startling mortality statistics would be conclusive evidence did any doubt exist. So long as the medical profession relegates its thinking to commercial men, the market will continue to be flooded with "baby foods" and the figures of infant mortality repeat themselves with sickening regularity. What, then, of a substitute for mother's milk? We have no substitute, as the death rate shows, but we have well. 